\title{
NUMERICAL SIMULATIONS OF A LOW MACH NUMBER MODEL IN HEAT EXCHANGER
}

\author{
Gloria Faccanoni ${ }^{1}$, Cédric Galusinski ${ }^{1}$ and Moustoifa Rafiou ${ }^{1}$ \\ ${ }^{1}$ Université de Toulon - IMATH, EA 2134, avenue de l'Université, 83957 La Garde, France
}

\begin{abstract}
This work is devoted to the numerical simulation of liquid-vapour flows in nuclear framework. We investigate a simplified model, named LMNC (for Low Mach Nuclear Core), describing the evolution of the coolant within a core of a Pressurized Water Reactor. This model, proposed in previous works, corresponds to the zero Mach asymptotic where the dilation of the fluid depends simply on the density of the fluid, function of the enthalpy. The goal of this work is to introduce a numerical approximation, validated on a reference test problem solved analytically, able to solve the $3 \mathrm{D}$ configuration.
\end{abstract}

\section{Introduction}

The modelling of nuclear reactors is hard to achieve since it requires the coupling of several multiscale multi-physics problems [6]. Indeed, a reactor is characterized by a large number of systems corresponding to different functions (heating, cooling, energy production, ... ). The natural process is thus to split the problems into lower-scale ones and then to carry out the coupling between them [11]. Moreover, there exist several industrial codes based on a compressible model providing numerical approximations of the whole nuclear reactor (see for instance [14, 4]).

A model was proposed in [7] to describe specifically flows within the reactor core in a simplified approach (which may be enriched to provide a more complete description of the overall process). Based on the assumption that the average Mach number is small, the model was derived through an asymptotic expansion performed in the compressible Navier-Stokes equations with an energy source term. This asymptotic approach amounts to filtering out the acoustic waves. Consequently, the mathematical nature of the resulting system of PDEs is modified which requires numerical methods that are different from the compressible Navier-Stokes framework. The model derived in [7] is called LmNC (for Low Mach Nuclear Core model) and consists of a transport equation upon a thermodynamic variable (here the total enthalpy), of a divergence constraint upon the velocity (with a nonlinear coupling source term which underlines the dilation property of the flow) and of the momentum equation.

In [2], for single-phase fluid with the stiffened gas equation of state (SG EOS), an explicit 1D unsteady solution was derived in the case of the power density $\Phi$ is constant and 1D academic numerical simulations were carried out relying on the method of characteristics.

Phase change was then taken into account in [3] where each pure phase was modelled through the SG EOS with tuned parameters. The modelling of the mixture phase based on thermodynamic equilibria is detailled in the aforementioned paper and turns out to be a phasewise SG EOS. Explicit 1D unsteady solutions can still be derived in the case of constant power densities. In the general case, 1D steady solutions are provided. The numerical scheme designed in [2] was adapted to phase change and applied to more physical tests.

The extension to dimension 2 was performed in [8] involving the software FreEFem ++ . The weak formulation was designed using the method of characteristics to handle the transport operators. In dimension 2, the issue consisted in dealing with the coupling of all equations in the system while in dimension 1, equations can be treated one after the other (in single phase flows).

However, although the aforementioned algorithm provided a relevant hint of the behaviour of the coolant fluid, discrepancies were noticed between numerical results (for instance values of the temperature at which mixture appears) and experimental values. That is why another approach was investigated. The analytical EOS was replaced by formulae fitting tabulated values provided in [13]. Hence each thermodynamic variable and each constitutive parameter were considered polynomial functions of the enthalpy and of the thermodynamic pressure in both liquid and vapour 
phases. The modelling of the mixture remains unchanged. This approach, presented in [9], shows a better assessment to experimental data. Moreover, to take into account the heat dissipation and the phase transition without introducing a level set function, a new weak formulation was designed (using again the method of characteristics to handle the transport operators).

In this paper, we present the extension to dimension 3 using a Finite Volume approach with staggered Cartesian grids on general geometries and a projection method to deal with the nonlinear dilation constraint. We use the phasewise SG EOS presented in [3] for simplicity.

\section{Setting of the mathematical problem}

The LMNC model is derived from the fully compressible Navier-Stokes equations expanding each variable into a power series of the Mach number $\mathcal{M}$ and taking the asymptotic limit for $\mathcal{M}$ going to zero. For each variable, the lowest order term remains in the equations, except for the pressure $p(t, \boldsymbol{x})$ which is split in two components: a thermodynamic pressure $p_{0}(t)$ uniform in space and a hydrodynamic pressure $\bar{p}(t, \boldsymbol{x})$. One has $p(t, \boldsymbol{x})=p_{0}(t)+\bar{p}(t, \boldsymbol{x})$. As the ratio $\bar{p}(t, \boldsymbol{x}) / p(t, \boldsymbol{x})=$ $\mathcal{O}\left(\mathcal{M}^{2}\right)$, the hydrodynamic pressure $\bar{p}(t, \boldsymbol{x})$ is much smaller than the thermodynamic pressure $p_{0}(t)$.

In this paper we take the assumption that pressure $p_{0}$ does not depend on time.

\subsection{Governing equations}

For some bounded domain $\Omega \subset \mathbb{R}^{3}$, the LMNC model reads

$$
\left\{\begin{array}{l}
\partial_{t} \varrho+\nabla \cdot(\varrho \mathbf{u})=0, \\
\partial_{t}(\varrho \mathbf{u})+\nabla \cdot(\varrho \mathbf{u} \otimes \mathbf{u}+\bar{p} \mathbb{I})=\nabla \cdot \sigma(\mathbf{u})+\varrho \mathbf{g}, \quad(t, \boldsymbol{x}) \in \mathbb{R}^{+} \times \Omega \\
\partial_{t}(\varrho h)+\nabla \cdot(\varrho h \mathbf{u})=\Phi(t, \boldsymbol{x}),
\end{array}\right.
$$

where $\mathbf{u}=(u, v, w)$ and $h$ denote respectively the velocity field and the total enthalpy of the fluid. The density $\varrho\left(h, p_{0}\right)$ is related to the enthalpy through an equation of state (EOS). The power density $\Phi(t, \boldsymbol{x})$ is a given function of time and space modelling the heating of the coolant fluid due to the fission reactions in the nuclear core. Finally, $\mathbf{g}=(0,-g, 0)$ is the gravity field and $\sigma(\boldsymbol{u})$ models viscous effects: the classic internal friction in the fluid, and also the friction on the fluid due to technological devices in the nuclear core (e.g. the friction on the fluid due to the fuel rods):

$$
\sigma(\mathbf{u})=\mu\left(h, p_{0}\right)\left(\nabla \mathbf{u}+(\nabla \mathbf{u})^{T}\right)+\eta\left(h, p_{0}\right)(\nabla \cdot \mathbf{u}) \mathbb{I},
$$

where $\mu\left(h, p_{0}\right)$ and $\eta\left(h, p_{0}\right)=-\frac{2}{3} \mu\left(h, p_{0}\right)$ are the viscosity coefficients determined by constitutive laws.

A 3D nonconservative formulation of the LMNC model reads

$$
\left\{\begin{array}{l}
\partial_{t} h+\mathbf{u} \cdot \nabla h=\frac{\Phi(t, \boldsymbol{x})}{\varrho\left(h, p_{0}\right)} \\
\partial_{t} \mathbf{u}+(\mathbf{u} \cdot \nabla) \mathbf{u}-\frac{1}{\varrho\left(h, p_{0}\right)} \nabla \cdot \sigma(\mathbf{u})+\frac{1}{\varrho\left(h, p_{0}\right)} \nabla \bar{p}=\mathbf{g}, \quad(t, \boldsymbol{x}) \in \mathbb{R}^{+} \times \Omega \\
\nabla \cdot \mathbf{u}=\frac{\beta\left(h, p_{0}\right)}{p_{0}} \Phi(t, \boldsymbol{x}),
\end{array}\right.
$$

where the compressibility coefficient $\beta\left(h, p_{0}\right)$ is defined as

$$
\beta(h, p) \stackrel{\text { def }}{=}-\left.\frac{p}{\varrho^{2}(h, p)} \cdot \frac{\partial \varrho}{\partial h}\right|_{p}(h, p) .
$$

We must also emphasize that model (2) is characterized by two pressure fields, which is classic in low Mach number approaches. The thermodynamic pressure $p_{0}$ is involved in the equation of state and is an average pressure (constant in time and space) within the core. The dynamic pressure $\bar{p}$ appears in the momentum equation $(2 \mathrm{~b})$ and can be considered as a perturbation around $p_{0}$. This pressure decomposition comes down to filtering out the acoustic waves which are no more involved in system (2). We mention that model (2) is only valid under the assumption that pressure $p_{0}$ does not depend on time. 


\begin{tabular}{cc}
\hline$h_{\ell}^{s}$ & $1.627 \times 10^{6} \mathrm{~J} \cdot \mathrm{K}^{-1}$ \\
$h_{g}^{s}$ & $3.004 \times 10^{6} \mathrm{~J} \cdot \mathrm{K}^{-1}$ \\
\hline$\varrho_{\ell}^{s}$ & $632.663 \mathrm{~kg} \cdot \mathrm{m}^{-3}$ \\
$\varrho_{g}^{s}$ & $52.937 \mathrm{~kg} \cdot \mathrm{m}^{-3}$ \\
\hline$T^{s}$ & $654.65 \mathrm{~K}$ \\
\hline
\end{tabular}

(a) Values at saturation for $p_{0}=15.5 \mathrm{MPa}$ deduced from the parameters of [12].

\begin{tabular}{cc}
\hline$\beta_{\ell}$ & $8.769 \times 10^{-3}$ \\
$\beta_{m}$ & $1.949 \times 10^{-1}$ \\
$\beta_{g}$ & $3.007 \times 10^{-1}$ \\
\hline$q_{\ell}$ & $-1.167 \times 10^{6} \mathrm{~J} \cdot \mathrm{K}^{-1}$ \\
$q_{m}$ & $1.501 \times 10^{6} \mathrm{~J} \cdot \mathrm{K}^{-1}$ \\
$q_{g}$ & $2.030 \times 10^{6} \mathrm{~J} \cdot \mathrm{K}^{-1}$ \\
\hline
\end{tabular}

(b) Orders of magnitude of the compressibility coefficient and the equivalent binding energy at $p_{0}=15.5 \mathrm{MPa}$.

Table 1: Parameters for phasewise Stiffened Gas Equation of State

\subsection{Equation of State: phasewise Stiffened Gas law}

We now have to build a single set of governing equations that can represent both phases, i.e. an equation of state (EOS) that relates the density $\varrho$ to the unknowns of the system (2). This relation yields the expression of the compressibility coefficient involved in equation (2c). The two phases in the mixture are assumed to be in thermodynamic equilibrium (of temperatures and chemical potentials) and mechanical equilibrium and the temperature in the mixture is taken to be equal to the saturation temperature. Consequently, the two-phase mixture is effectively treated as a single (pseudo) fluid whose properties are suitable averages of the phasic properties of the individual phases. The balance equations are the same as those for the single-phase flow as shown in [3]; but each primary variable now represents the state of a homogeneous mixture of two phases. Thus the stiffened gas law for two-phase flow with phase transition reads:

$$
\varrho\left(h, p_{0}\right)=\frac{p_{0} / \beta\left(h, p_{0}\right)}{h-q\left(h, p_{0}\right)}
$$

with $\beta\left(h, p_{0}\right)$ and $q\left(h, p_{0}\right)$ phasewise constant and defined by:

$$
\beta\left(h, p_{0}\right)=\left\{\begin{array}{ll}
\beta_{\ell}\left(p_{0}\right), & \text { if } h \leq h_{\ell}^{s}\left(p_{0}\right), \\
\beta_{m}\left(p_{0}\right), & \text { if } h_{\ell}^{s}\left(p_{0}\right)<h<h_{g}^{s}\left(p_{0}\right), \\
\beta_{g}\left(p_{0}\right), & \text { if } h \geq h_{g}^{s}\left(p_{0}\right),
\end{array} \quad q\left(h, p_{0}\right)= \begin{cases}q_{\ell}, & \text { if } h \leq h_{\ell}^{s}(p), \\
q_{m}\left(p_{0}\right), & \text { if } h_{\ell}^{s}\left(p_{0}\right)<h<h_{g}^{s}\left(p_{0}\right), \\
q_{g}, & \text { if } h \geq h_{g}^{s}\left(p_{0}\right) .\end{cases}\right.
$$

In Table 1 values for water and steam computed with parameters of [3].

\subsection{Boundary conditions}

Boundary conditions $(\mathrm{BC})$ are specified in $3 \mathrm{D}\left(\Omega=\left[0, L_{x}\right] \times\left[0, L_{y}\right] \times\left[0, L_{z}\right]\right.$ where the vertical variable is $y$ ).

- The fluid is injected vertically at the bottom of the core $(y=0)$ at a given enthalpy $h_{e}$ and at a given vertical flow rate $D_{e}$ :

$$
\left\{\begin{array}{l}
h(t, x, 0, z)=h_{e}(t, x, z) \\
(\varrho \mathbf{u})(t, x, 0, z)=\left(0, D_{e}(t, x, z), 0\right) .
\end{array}\right.
$$

The entrance velocity $v_{e}(t, x, z)$ to apply at $y=0$ is deduced from the relation $v_{e}(t, x, z)=$ $D_{e}(t, x, z) / \varrho_{e}(t, x, z)$ where $\varrho_{e} \stackrel{\text { def }}{=} \varrho\left(h_{e}, p_{0}\right)$.

An alternative consists in prescribing both density and vertical flow rate:

$$
\begin{cases}\varrho(t, x, 0, z) & =\varrho_{e}(t, x, z), \\ (\varrho \boldsymbol{u})(t, x, 0, z) & =\left(0, D_{e}(t, x, z), 0\right) .\end{cases}
$$

In that case, we need to invert the EOS to compute $h_{e}$ from $\varrho_{e}$. 
- At the top of the core, we consider a free outflow which is not detailed here, see for example [5] for a well-posed closure. The approach chosen here can be detailed after introducing time discretization and splitting techniques in the manner of [10].

- On the lateral walls, upon the velocity we consider slip or no slip boundary conditions. Slip coditions are used for 1D solutions.

\subsection{Assumptions}

For the problem to be well-posed, we impose some assumptions upon the data and the initial/boundary states:

(i) $\Phi(t, \boldsymbol{x})$ is nonnegative for all $(t, \boldsymbol{x}) \in \mathbb{R}^{+} \times \Omega$, characterizing the fact that we study a nuclear core where the coolant fluid is heated;

(ii) $p_{0}$ is a positive constant;

(iii) $D_{e}(t, x, z)>0$ for all $t \geq 0$ and $(x, z) \in\left[0, L_{x}\right] \times\left[0, L_{z}\right]$ (the flow is upward);

(iv) $h_{\text {init }}$ is such that $h_{\text {init }}(x, 0, z)=h_{e}(t=0, x, z)$ for all $(x, z) \in\left[0, L_{x}\right] \times\left[0, L_{z}\right]$;

(v) $\mathbf{u}_{\text {init }}$ is such that

$$
\left\{\begin{array}{l}
\nabla \cdot \mathbf{u}_{\text {init }}=\frac{\beta\left(h, p_{0}\right)}{p_{0}} \Phi(0, \boldsymbol{x}), \\
\mathbf{u}_{\text {init }}(x, 0, z)=\mathbf{u}_{e}(t=0, x, z),
\end{array}\right.
$$

corresponding to the fact that the steady equation (2c) is initially satisfied, which means that initial conditions are well-prepared (see [3] for instance);

\subsection{Steady state solution}

In dimension 1, we can explicitly compute steady solutions of the LMNC model no matter what the equation of state $[3,9]$.

Proposition 1 (Steady states). We consider the $1 D$ steady case, i.e. $\varrho_{e}, D_{e}$ and $\Phi$ do not depend on time. Then, System (1) admits a unique steady solution given by

$$
h(y)=h_{e}+\frac{1}{D_{e}} \int_{0}^{y} \Phi(z) \mathrm{d} z, \quad v(y)=\frac{D_{e}}{\varrho\left(h(y), p_{0}\right)} .
$$

A distinctive feature of the LMNC model is that the steady enthalpy does not depend on the equation of state except through the computation of $h_{e}$. Moreover, given the expression of $h$ in Proposition 1, it can be stated whether the steady fluid appears only as a (pure) liquid phase (if $\left.h(y)<h_{\ell}^{s}\right)$ or as a mixture $\left(h_{\ell}^{s} \leq h(y) \leq h_{g}^{s}\right)$ or also as a (pure) vapour phase $\left(h(y)>h_{g}^{s}\right)$. We define $y_{\ell}^{s}$ and $y_{g}^{s}$ as the solutions of the equations $h(y)=h_{\ell}^{s}$ and $h(y)=h_{g}^{s}$ respectively.

Note that with phasewise SG EOS, we can compute the transient $1 \mathrm{D}$ solution when $\Phi$ is constant [3].

\section{Numerical scheme}

The spatial discretization of the model is based on finite volumes for staggered grid, where the dynamic pressure is located at the center of the cells, while the components of the velocity densities and the enthalpy are located on the faces. This choice is motivated by the fluid model looking like incompressible flows. This choice is then conserved for the enthalpy equation.

Here we detail only the time discretization. In order to avoid complex coupling between the enthalpy equation (2a) and the generalized Navier-Stokes equation (2b)-(2c), the system is 
decoupled as follows,

$$
\left\{\begin{array}{l}
\partial_{t} h+\mathbf{u} \cdot \nabla h=\frac{\Phi(t, \boldsymbol{x})}{\varrho\left(h, p_{0}\right)} \\
\partial_{t} \mathbf{u}+(\mathbf{u} \cdot \nabla) \mathbf{u}-\frac{1}{\varrho\left(\bar{h}, p_{0}\right)} \nabla \cdot \sigma(\mathbf{u})+\frac{1}{\varrho\left(\bar{h}, p_{0}\right)} \nabla \bar{p}=\mathbf{g} \\
\nabla \cdot \mathbf{u}=\frac{\beta\left(\bar{h}, p_{0}\right)}{p_{0}} \Phi(t, \boldsymbol{x})
\end{array}\right.
$$

where $\bar{h}(t, \boldsymbol{x}) \stackrel{\text { def }}{=} h(t-\delta t, \boldsymbol{x})$ is then known when solving $(6 \mathrm{~b})-(6 \mathrm{c})$ on a time step $\delta t$. It then allows to solve the decoupled equation (6a), on a time step, with a given transport velocity $\mathbf{u}$. The system (6b)-(6c) is solved thanks to classical technique used for incompressible and inhomogeneous flows. This part is detailed in appendix. We focus here on the enthalpy equation (6a) driven by a transport term with a dilatable flow and a nonlinear source term. This equation is written as follows

$$
\partial_{t} h=-\nabla \cdot(\mathbf{u} h)+h \nabla \cdot \mathbf{u}+\frac{\Phi(t, \boldsymbol{x})}{\varrho\left(h, p_{0}\right)},
$$

so that the three terms of the right hand-side are approached thanks to a time Strang splitting, leading to an order 2 approximation with respect to time. The first term of the right hand-side is discretized with an explicite order 2 scheme with respect to time and space. The second term is solved thanks to the exact solution and the third term is discretized with an explicite order 2 scheme with respect to time.

In the following section, we discuss the validation of these approximations thanks to the exact 1D solution asymptotic steady state solution of Proposition 1.

\section{Numerical Tests}

For any test, reference values for pressure and gravity intensity are $p_{0}=155 \times 10^{5} \mathrm{~Pa}$ and $g=$ $9.81 \mathrm{~m} \cdot \mathrm{s}^{-2}$. The viscosity is constant in time and space (the same for both phases): $\mu\left(h, p_{0}\right)=$ $8.4 \times 10^{-1} \mathrm{~kg} \cdot \mathrm{m}^{-1} \cdot \mathrm{s}^{-1}, \eta\left(h, p_{0}\right)=-\frac{2}{3} \mu\left(h, p_{0}\right)$. The reactor is a box $\Omega=\left\{\left[0, L_{x}\right] \times\left[0, L_{y}\right] \times\left[0, L_{z}\right]\right\}$ with $L_{x}=L_{z}=1 \mathrm{~m}$ and $L_{y}=4.2 \mathrm{~m}$.

Power density Constant in time

$$
\Phi(t, x, y, z)= \begin{cases}170 \times 10^{6} \mathrm{~W} \cdot \mathrm{m}^{-3} & \text { for all } y>\frac{1}{2} \\ 0 \mathrm{~W} \cdot \mathrm{m}^{-3} & \text { otherwise }\end{cases}
$$

Let us denote $\hat{\Phi}_{\kappa} \stackrel{\text { def }}{=} \beta_{\kappa} \Phi / p_{0}$, thus

$$
\hat{\Phi}_{\ell}=0.096 \mathrm{~s}^{-1}, \quad \hat{\Phi}_{m}=2.137 \mathrm{~s}^{-1}, \quad \hat{\Phi}_{g}=3.297 \mathrm{~s}^{-1} .
$$

BC-bottom Inflow data: liquid phase constant in time and space

$$
\begin{aligned}
\mathbf{u}_{e}(t, x, z) & =\left(u_{e}(t, x, z), v_{e}(t, x, z), w_{e}(t, x, z)\right)=(0,0.4,0) \mathrm{m} \cdot \mathrm{s}^{-1} \\
\varrho_{e}(t, x, z) & =749 \mathrm{~kg} \cdot \mathrm{m}^{-3}, \\
h_{e}\left(\varrho_{e}(t, x, z)\right) & =h_{\ell}\left(\varrho_{e}(t, x, z)\right)=\frac{p_{0} / \beta_{\ell}}{\varrho_{e}(t, x, z)}+q_{\ell} \simeq 1.193 \times 10^{6} \mathrm{~J} \cdot \mathrm{kg}^{-1} .
\end{aligned}
$$

Expected results The domain is initially filled with liquid. Mixture appears for $y_{\ell}^{s}<y<y_{g}^{s}$ and pure vapour appears for $y>y_{g}^{s}$ where

$$
y_{\ell}^{s}=\frac{1}{2}+\frac{\varrho_{e} v_{e}}{\Phi}\left(h_{\ell}^{s}-h_{e}\right) \simeq \frac{1}{2}+0.765 \mathrm{~m}, \quad y_{g}^{s}=\frac{1}{2}+\frac{\varrho_{e} v_{e}}{\Phi}\left(h_{\mathrm{g}}^{s}-h_{e}\right) \simeq \frac{1}{2}+3.192 \mathrm{~m}
$$


The asymptotic state is given by (see Figure 1):

$$
\begin{aligned}
& \mathbf{u}^{\infty}(x, y, z)=\left(u^{\infty}(x, y, z), v^{\infty}(x, y, z), w^{\infty}(x, y, z)\right) \\
& u^{\infty}(x, y, z)=0 \\
& v^{\infty}(x, y, z)= \begin{cases}v_{e}+\hat{\Phi}_{\ell}\left(y-\frac{1}{2}\right) & \text { for all } y_{\ell}^{s}>y>\frac{1}{2} \\
v_{e}+\hat{\Phi}_{\ell}\left(y_{\ell}^{s}-\frac{1}{2}\right)+\hat{\Phi}_{m}\left(y-y_{\ell}^{s}\right) & \text { for all } y_{g}^{s}>y>y_{\ell}^{s} \\
v_{e}+\hat{\Phi}_{\ell}\left(y_{\ell}^{s}-\frac{1}{2}\right)+\hat{\Phi}_{m}\left(y_{g}^{s}-y_{\ell}^{s}\right)+\hat{\Phi}_{g}\left(y-y_{g}^{s}\right) & \text { for all } y>y_{g}^{s} \\
v_{e} & \text { otherwise }\end{cases} \\
& w^{\infty}(x, y, z)=0, \\
& h^{\infty}(x, y, z)= \begin{cases}h_{e}+\frac{\Phi}{\varrho_{e} v_{e}}\left(y-\frac{1}{2}\right), & \text { for all } y>\frac{1}{2} \\
h_{e} & \text { otherwise. }\end{cases}
\end{aligned}
$$

The analytic expression of dynamic pressure can be computed a posteriori.

\section{Comparisons of numerical and exact solutions}

- In Figure 1, we show the exact asymptotic solution with a velocity linear by parts. The slope changes occurs with phase changes and are significantly different. Thereby, a slight modification on the enthalpy modify the position of change phase $y_{\ell}^{s}$ and $y_{g}^{s}$ shown on the figure and the velocity is significantly modified due to strong differences between $\hat{\Phi}_{l}, \hat{\Phi}_{m}$ and $\hat{\Phi}_{g}$.

- On the Figure 2a, equation (6a) is solved with the order 2 scheme proposed above but for a fixed given velocity corresponding to the exact asymptotic velocity (11). It leads to a convergent approximation with an under-estimated numerical solution. This suggests to introduce a scheme able to catch the asymptotic solution with respect to time. The time order 2 scheme used for the conservative term of (7) is a Runge-Kutta scheme corresponding to a $\theta$-scheme with $\theta=\frac{1}{2}$ whose first order 1 prediction under-estimates whereas the second prediction over-estimates in the manner of an implicit scheme. We then introduce a $\theta$-scheme with $\theta=0.3$ (the weight on the first prediction) leading to a better numerical solution on coarsed grid. Such a solution is plotted on the Figure $2 \mathrm{~b}$.

- Then, the two schemes are compared for the full model (6). The advantage of the tuned scheme is not conserved due to perturbations induced on the velocity. The order 2 scheme reveals better for the full model as shown on the Figure $2 \mathrm{c}$ and $2 \mathrm{~d}$. This conclusion is condensed on the Figure 3 for the grid with 200 cells in $y$ direction.

- On the velocity graphs shown on the Figure 4, the divergence of the velocity (8) can be observed as the slope of curves and is precisely approached. Nevertheless, errors on phase change positions induce error on velocity which influence the enthalpy. This is why the order 2 scheme is better on the full model whereas the tuned model is more appropriated for the enthalpy equation. The convergence with respect to the grid size for the full model is also visible on the velocity.

\section{Conclusion and Perspectives}

The LMNC model introduces a strong coupling between the Navier-Stokes equations and the enthalpy equation since the dilation of the fluid depends on the enthalpy transported by the flow. Perturbations on velocities induces sensitive perturbations on the enthalpy and then on regions of phase change. Nevertheless, some auto-corrections of perturbations occur since excessive enthalpies induce sooner phase change leading to excessive velocities. The increased velocities cools the flow and then reduces the excessive enthalpy.

With our 3D code, the presented results for 1D exact asymptotic solutions show convergent results as well for decoupled problem as for the full LMNC code.

Previous works with different numerical strategy was already performed for the LMNC model, but this one is the first produced on staggered grids with projection method inside a $3 \mathrm{D}$ code, allowing realistic simulations. 


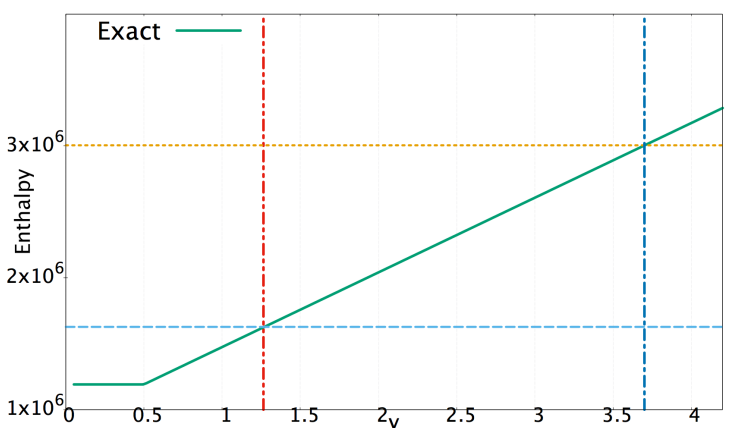

(a) Enthalpy.

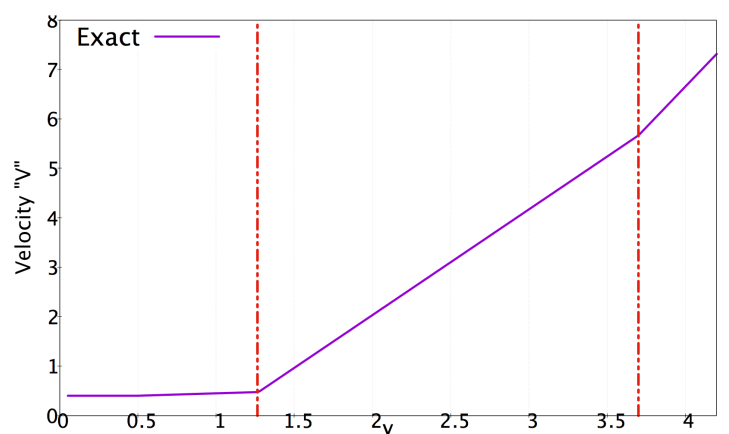

(b) Velocity.

Figure 1: Exact asymptotic solution.

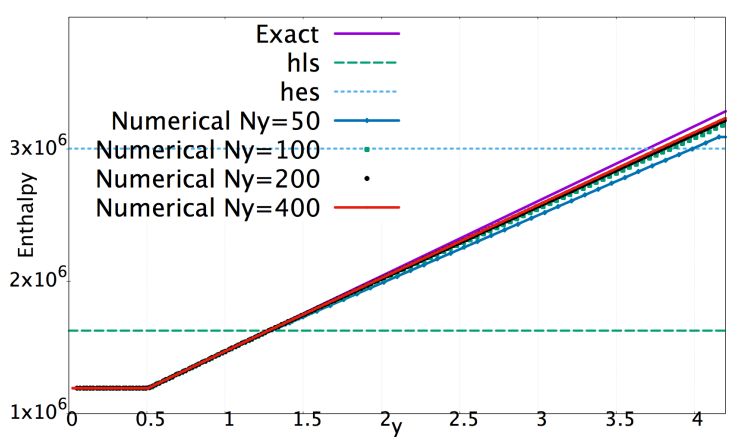

(a) Enthalpy with $v$ computed analytically and equation (7) solved by order 2 scheme

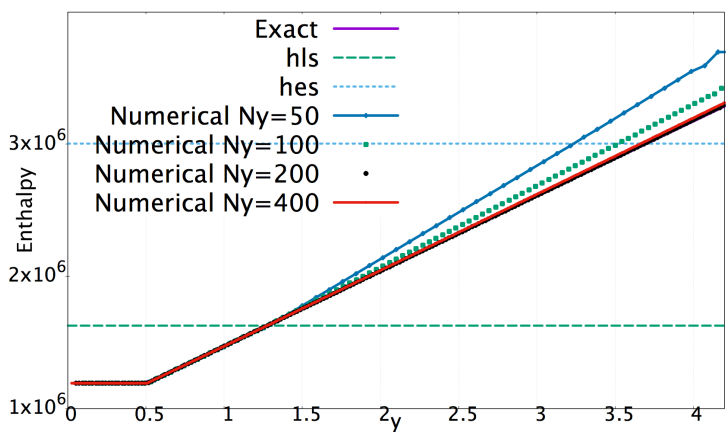

(c) Enthalpy solved by the full model with order 2 scheme

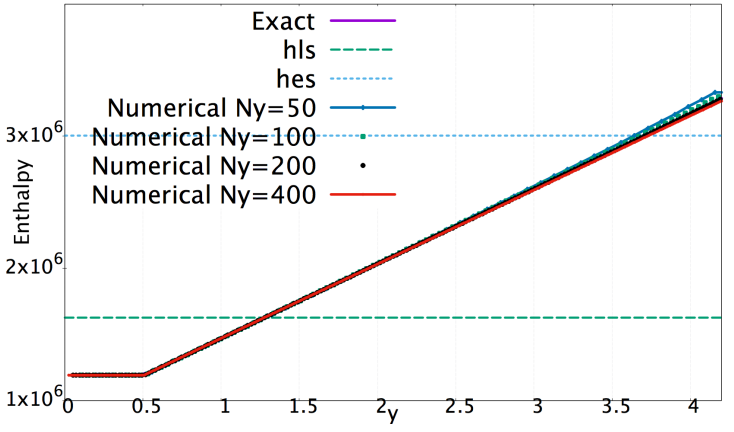

(b) Enthalpy with $v$ computed analytically and equation (7) solved by tuned order 1 scheme

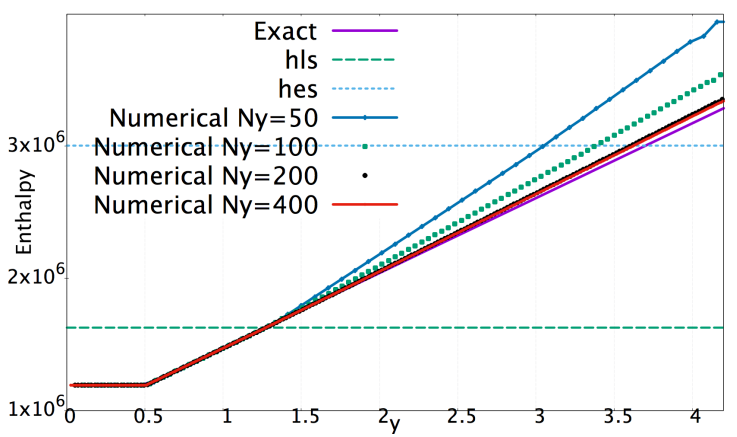

(d) Enthalpy solved by the full model with the tuned order 1 scheme

Figure 2: Enthalpy with different grids. 


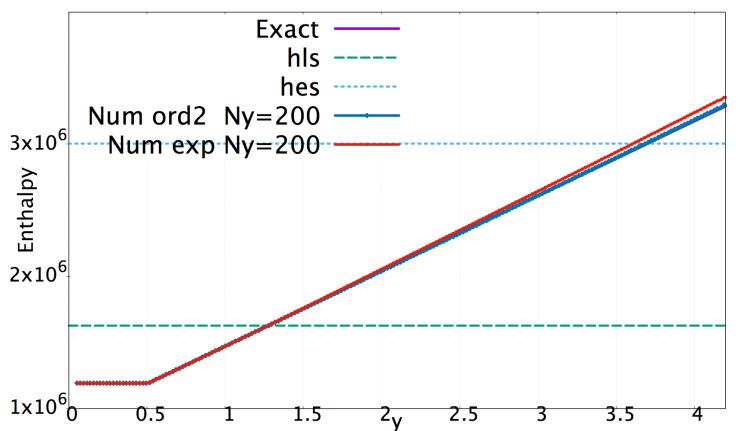

(a) Enthalpy with $v$ computed analytically and equation (7) solved by order 2 scheme vs tuned order 1 scheme

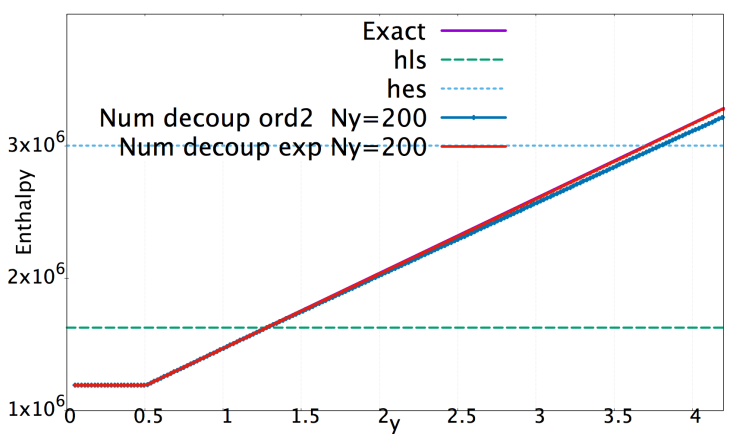

(b) Enthalpy solved by the full model with order 2 scheme vs tuned order 1 scheme

Figure 3: Comparisons of the two schemes on the equation (7). We show the enthalpy computed with 200 cells in $y$ direction.

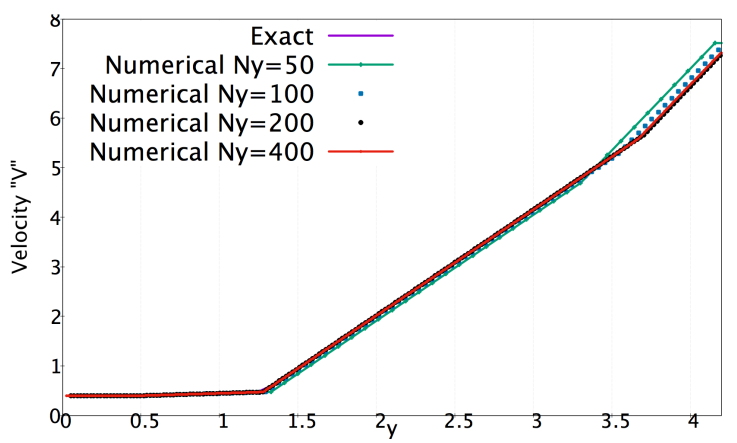

(a) Velocity with equation (7) solved by order 2 scheme

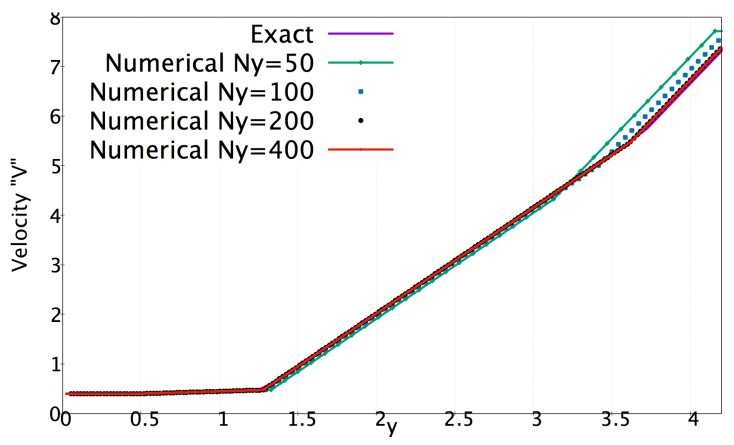

(b) Velocity with equation (7) solved by tuned order 1 scheme

Figure 4: Velocity for different grids. 
Other numerical strategies have to be tested in order to try to reduce the space discretization leading to converged results. Namely, the continuity equation on the density has to be solved instead of enthalpy equation.

A more distant goal concerns the comparison between the compressible original model and its asymptotic, that is the LMNC model studied here.

Acknowledgment This work was partially funded by the CNRS project call NEEDS (nuclear, energy, environment, waste and society) and "Conseil départemental" of Var (France).

\section{Appendix: Fluid solver discretization}

The viscous and buoyancy forces enter the computation of the dynamic pressure in low-Machnumber flow in a similar way as they enter the pressure computation in incompressible flow, except for a nonzero velocity-divergence constraint. The convection operator is discretized explicitly. The diffusion is treated implicitly to avoid the stability constraint on the time step induced by the second-order diffusion operator. The pressure and velocity are decoupled to avoid solving the coupled saddle-point problem. This decoupling is done by using a classical prediction-projection method.

The solution of equations $(6 \mathrm{~b})$ and $(6 \mathrm{c})$ for $\left(\mathbf{u}^{n+1}, \bar{p}^{n+1}\right)$ is performed by a so-called pressurecorrection method. These schemes consist of two substeps per (fictive) time step $k$ : the pressure is treated explicitly in the first substep and corrected in the second substep by projecting the intermediate velocity onto the space of "divergence-fixed" field. We repeat this two substeps until the divergence of $\mathbf{u}^{n+1}$ is equal to $\frac{\beta^{n+1}}{p_{0}} \Phi^{n+1}(\boldsymbol{x})$.

Let $k=0$ and $\bar{p}^{n, k}=\bar{p}^{n}$. While $\left\|\nabla \cdot \mathbf{u}^{n+1, k}-\frac{\beta^{n+1}}{p_{0}} \Phi^{n+1}\right\|>$ TOLL repeat:

[Velocity predictor] For an intermediate velocity $\widetilde{\mathbf{u}}^{n+1, k}$, solve the semi-implicit equation

$$
\frac{\varrho^{n+1}}{\Delta t} \widetilde{\mathbf{u}}^{n+1, k}-\nabla \cdot \sigma\left(\widetilde{\mathbf{u}}^{n+1, k}\right)=\varrho^{n+1}\left(\frac{1}{\Delta t} \mathbf{u}^{n}-\left(\mathbf{u}^{n} \cdot \nabla\right)\right) \mathbf{u}^{n}+\varrho^{n+1} \mathbf{g}-\nabla \bar{p}^{n} .
$$

Supposing that $\widetilde{\mathbf{u}}^{n+1, k}$ verifies the divergence constraint (6c), and if $\nabla \mu^{n+1}$ can be neglected (and then $\nabla \eta^{n+1}$ also), i.e. $\mu^{n+1}=\mu$ for all $n$, then we have

$$
\begin{aligned}
\nabla \cdot \sigma\left(\widetilde{\mathbf{u}}^{n+1, k}\right) & \simeq \mu \Delta \widetilde{\mathbf{u}}^{n+1, k}+(\mu+\eta) \nabla\left(\frac{\beta^{n+1}}{p_{0}} \Phi^{n+1}(\boldsymbol{x})\right) \\
& =\mu\left(\Delta \widetilde{\mathbf{u}}^{n+1, k}+\frac{4}{3} \nabla\left(\frac{\beta^{n+1}}{p_{0}} \Phi^{n+1}(\boldsymbol{x})\right)\right)
\end{aligned}
$$

so that each component of the velocity can be computed independently of the others as follows:

$$
\frac{\varrho^{n+1}}{\mu \Delta t} \widetilde{\mathbf{u}}^{n+1, k}-\Delta \widetilde{\mathbf{u}}^{n+1, k}=\frac{4}{3} \nabla\left(\frac{\beta^{n+1}}{p_{0}} \Phi^{n+1}(\boldsymbol{x})\right)+\frac{\varrho^{n+1}}{\mu \Delta t} \mathbf{u}^{n}+\frac{\varrho^{n+1}}{\mu}\left(\mathbf{g}-\left(\mathbf{u}^{n} \cdot \nabla\right) \mathbf{u}^{n}\right)-\frac{1}{\mu} \nabla \bar{p}^{n} .
$$

[Pressure correction] Once $\widetilde{\mathbf{u}}^{n+1, k}$ is known, the projection step consists in extracting $\mathbf{u}^{n+1, k}$ such a way that $\nabla \cdot \mathbf{u}^{n+1, k}$ satisfies divergence constraint (16),

$$
\left\{\begin{array}{l}
\nabla \cdot \mathbf{u}^{n+1, k}=\frac{\beta^{n+1}}{p_{0}} \Phi^{n+1}(\boldsymbol{x}), \\
\frac{\mathbf{u}^{n+1, k}-\widetilde{\mathbf{u}}^{n+1, k}}{\Delta t}+\frac{1}{\varrho^{n+1}} \nabla \widetilde{\bar{p}}^{n+1, k}=\mathbf{0} .
\end{array}\right.
$$

Taking the divergence of the second equation and enforcing the first equation leads to

$$
\begin{cases}\nabla \cdot\left(\frac{\nabla \widetilde{\bar{p}}^{n, k}}{\varrho^{n+1}}\right)=\frac{1}{\Delta t}\left(\nabla \cdot \widetilde{\mathbf{u}}^{n+1, k}-\frac{\beta^{n+1}}{p_{0}} \Phi^{n+1}(\boldsymbol{x})\right), & \\ \partial_{\mathbf{n}} \widetilde{\bar{p}}^{n, k}=K(x), & \text { for } \boldsymbol{x} \in \partial \Omega\end{cases}
$$


where $\widetilde{\bar{p}}^{n, k}=\bar{p}^{n, k}-\bar{p}^{n}$ is the pressure increment and the function $K$ is zero on the boundary except for the outflow boundary where $K$ is the constant satisfying the compatibility condition between the boundary condition and the source term of (17).

[Updates] Once the dynamic pressure is obtained, the velocity $\mathbf{u}^{n+1, k+1}$ is calculated from

$$
\mathbf{u}^{n+1 k+1}=\widetilde{\mathbf{u}}^{n+1, k+1}-\frac{\Delta t}{\varrho^{n+1}} \nabla \widetilde{\bar{p}}^{n, k}
$$

Finally the dynamical pressure $\bar{p}^{n, k+1}$ is updated as follows (this step is optional)

$$
\bar{p}^{n, k+1}=\bar{p}^{n, k}+\widetilde{\bar{p}}^{n, k+1} .
$$

When $\left\|\nabla \cdot \mathbf{u}^{n+1, k+1}-\frac{\beta^{n+1}}{p_{0}} \Phi^{n+1}\right\| \leq$ TOLL, we set $\mathbf{u}^{n+1}=\mathbf{u}^{n+1, \infty}$ and $\bar{p}^{n+1}=\bar{p}^{n, \infty}$.

\section{References}

[1] S. Benteboula. "Solving Navier-Stokes equations at low Mach number. Application to the variable density vortex rings." Theses. Université Paris-Est, Dec. 2006.

[2] M. Bernard, S. Dellacherie, G. Faccanoni, B. Grec, O. Lafitte, T.-T. Nguyen, and Y. Penel. "Study of low Mach nuclear core model for single-phase flow". In: ESAIM Proc. 38 (2012), pp. 118-134.

[3] M. Bernard, S. Dellacherie, G. Faccanoni, B. Grec, and Y. Penel. "Study of a low Mach nuclear core model for two-phase flows with phase transition I: stiffened gas law". In: ESAIM: Mathematical Modelling and Numerical Analysis 48 (2014), pp. 1639-1679.

[4] D. Bestion. "The physical closure laws in the CATHARE code". In: Nucl. Eng. Des. 124.3 (1990), pp. 229-245.

[5] C.-H. Bruneau and P. Fabrie. "New efficient boundary conditions for incompressible NavierStokes equations: a well-posedness result". In: ESAIM: Mathematical Modelling and Numerical Analysis 30.7 (1996), pp. 815-840.

[6] J.M. Delhaye. Thermohydraulique des réacteurs. EDP sciences, 2008.

[7] S. Dellacherie. "On a low Mach nuclear core model". In: ESAIM Proc. 35 (2012), pp. 79-106.

[8] S. Dellacherie, G. Faccanoni, B. Grec, E. Nayir, and Y. Penel. "2D numerical simulation of a low Mach nuclear core model with stiffened gas using FreeFem++". In: ESAIM: Proceedings 45 (2014), pp. 138-147.

[9] S. Dellacherie, G. Faccanoni, B. Grec, and Y. Penel. "Accurate steam-water equation of state for two-phase flow LMNC model with phase transition". 2018.

[10] C. Galusinski, C. Mazoyer, S. Meradji, A. Molcard, and Y. Ourmières. "Inlet and outlet open boundary conditions for incompressible Navier-Stokes equations". In: Proceedings Topical Problems of Fluid Mechanics 2017 (2017), pp. 139-146.

[11] O. Hurisse. "Couplage interfacial instationnaire de modèles diphasiques". PhD thesis. Univ. Aix-Marseille I, 2006.

[12] O. Le Métayer, J. Massoni, and R. Saurel. "Elaborating equations of state of a liquid and its vapor for two-phase flow models". French. In: Int. J. Therm. Sci. 43.3 (2004), pp. 265-276.

[13] E. W. Lemmon, M. O. McLinden, and D. G. Friend. Thermophysical Properties of Fluid Systems. National Institute of Standards and Technology, Gaithersburg MD, 20899.

[14] TRACE V5.0 Theory Manual, Field Equations, Solution Methods and Physical Models. Tech. rep. U.S. Nuclear Regulatory Commission, 2008. 